\title{
Ultrasonically driven nanomechanical single-electron shuttle
}

\author{
DANIEL R. KOENIG*, EVA M. WEIG AND JORG P. KOTTHAUS \\ Center for NanoScience and Fakultät für Physik der Ludwig-Maximilians-Universität, Geschwister-Scholl-Platz 1, 80539 München, Germany \\ *e-mail: daniel.koenig@physik.uni-muenchen.de
}

The single-electron transistor is the fastest and most sensitive electrometer available today ${ }^{1,2}$. Single-electron pumps and turnstiles are also being explored as part of the global effort to redefine the ampere in terms of the fundamental physical constants $^{3-6}$. However, the possibility of electrons tunnelling coherently through these devices, a phenomenon known as co-tunnelling ${ }^{7}$, imposes a fundamental limit on device performance. It has been predicted ${ }^{8}$ that it should be possible to completely suppress co-tunnelling in mechanical versions of the single-electron transistor', which would allow mechanical devices to outperform conventional single-electron transistors in many applications. However, the mechanical devices developed so far are fundamentally limited by unwanted interactions with the electrical mechanisms that are used to excite the devices ${ }^{10-12}$. Here we show that it is possible to overcome this problem by using ultrasonic waves rather than electrical currents as the excitation mechanism, which we demonstrate at low temperatures. This is a significant step towards the development of high-performance devices.

The mechanical single-electron transistors (MSETs) we fabricate are nanomechanical electron shuttles with a back gate, which is not used in the work presented here. The MSETs consist of a freely suspended silicon nitride string (Fig. la,b). The string has a length of $14 \mu \mathrm{m}$, width of $70 \mathrm{~nm}$ and height of $100 \mathrm{~nm}$. A gold island with dimensions of $\sim 140 \mathrm{~nm}$ (length), $\sim 170 \mathrm{~nm}$ (width) and $\sim 60 \mathrm{~nm}$ (height) is located at the centre of the string. The silicon nitride string can be excited so that the gold island oscillates between the source and drain electrodes, which are placed within a distance of $80 \mathrm{~nm}$ on either side of the island (Fig. 1a). If a voltage $V_{\text {sd }}$ is applied between source and drain, the oscillating island can transport electrons from one electrode to the other. The gold structures located symmetrically on the string to the left and right of the island in Fig. 1a are weights to tune the eigenfrequency of the MSET. Figure 1c shows an array of 44 MSETs. All structures are connected in parallel between two interdigitated comb electrodes that form the source and drain. Every MSET has its unique eigenfrequency realized by individually customized weights. This allows for large-scale integration of independently controllable devices, as each MSET can be selectively addressed by its characteristic eigenfrequency. We chose silicon nitride with a tensile stress of $1.38 \mathrm{GPa}$ as the resonator material. This material allows one of the highest mechanical quality factors in nanoelectromechanical systems to be achieved ${ }^{13}$.

Actuation of the MSETs is realized by applying ultrasonic waves, which are produced using a piezo actuator. By placing the sample chip inside a Faraday cage with solid 3-mm titanium walls (Fig. 2a,b), the sample is perfectly shielded from the electrical signal required for the piezo actuation. This is a key feature, as it allows for absolutely undisturbed MSET operation. The chip is glued to the inside of the cage wall. The source and drain are electrically contacted to coaxial cables, which are fed through the side walls of the cage. The piezo is mechanically pressed to the outer side of the wall to which the sample is attached. With this configuration the ultrasonic waves are transmitted through the cage wall as well as through the sample substrate to drive the MSETs. The mechanical resonances of the MSETs can be probed using a scanning electron microscope $(\mathrm{SEM})^{14}$ as illustrated in Fig. 3a,b without and with actuation, respectively. We determined the eigenfrequencies of the MSETs of the array in Fig. 1c to lie in the range between 3.5 and $6 \mathrm{MHz}$.

The experimental configuration for electrical transport measurements is illustrated in Fig. 2a. It allows the measurement of the time-averaged current $\overline{I_{\mathrm{sd}}}$ across the island with a resolution corresponding to just $1 / 20$ of an electron per shuttle period. The Faraday cage is placed within an evacuated sample tube (Attocube AG), which can be lowered into a helium dewar. The electrical transport measurement shown in Fig. 3c was conducted at a temperature of $\sim 20 \mathrm{~K}$ and a helium exchange gas pressure of $7.5 \times 10^{-4} \mathrm{mbar}$, and was corrected for a small offset of the current-voltage amplifier. The plot shows the transport resonance spectrum of the MSET array for an actuation frequency $f_{\text {drive }}$ between $3.5 \mathrm{MHz}$ and $6 \mathrm{MHz}$. The driving power $P_{\text {drive }}$ of the signal generator was $25 \mathrm{dBm}$ and $V_{\text {sd }}=0.8 \mathrm{~V}$. Eight MSETs of the array show resonances in the transport measurement. This demonstrates that not all of the vibrating MSETs give rise to charge transport, although inspection with an electron microscope after the experiments confirmed the functionality of all the MSETs. The reason for this is that our mechanical resonators can be classified as so-called impacting systems ${ }^{15,16}$. These deceptively simple systems have been shown to display complicated subharmonic and chaotic behaviour. The mechanical complexity of the MSETs is caused by their nonlinear nature, which is imposed by the side electrodes constituting the impacting boundary conditions. This means that charge transport by mechanically excited MSETs is not necessarily limited only by the islands' electrical capacitance, but also by their complex mechanical response. Another consequence of the system's nonlinearity is that the resonance curves generally do not follow a Lorentzian line shape. This is illustrated in Fig. 3d, which shows a high-resolution frequency sweep for the MSET 

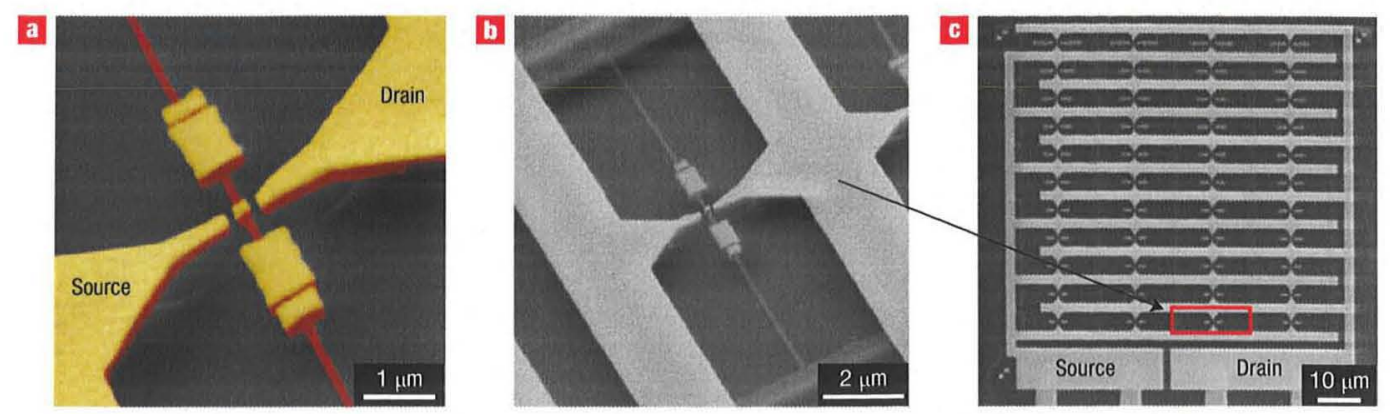

Figure 1 The MSET devices. a, A false-colour SEM image taken at an angle to reveal the three-dimensional character of the MSET. A gold (yellow) island is located at the centre of a doubly clamped freely suspended silicon nitride (red) string. The gold island can shuttle electrons between the source and drain electrodes when excited by ultrasonic waves. b. An SEM image taken at an angle showing a complete MSET together with the clamping points of the freely suspended string. c, An SEM image showing a top view of an array of 44 MSETs, which are connected in parallel between two interdigitated comb electrodes.
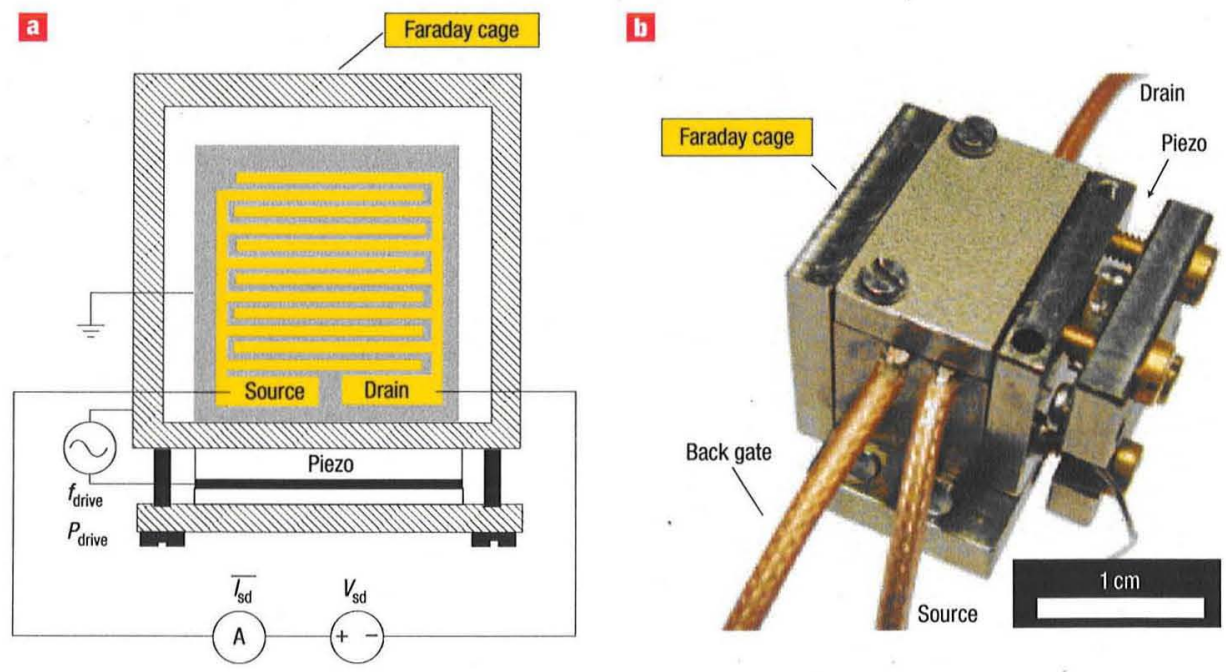

Figure 2 The Faraday cage and piezo actuation system. a, Simplified experimental setup with sample chip inside the Faraday cage (schematic not to scale). The piezo for actuation is pressed against the cage wall from the outside. b. Photograph of the Faraday cage together with the piezo actuation unit.

corresponding to the third resonance in Fig. $3 \mathrm{c}$ at a driving power $P_{\text {drive }}=27.5 \mathrm{dBm}$ and a source-drain voltage $V_{\text {sd }}=-2 \mathrm{~V}$.

The above discussion demonstrates successful resonant charge transport as function of $f_{\text {drive }}$. We now investigate charge transport as a function of $V_{\mathrm{sd}}$ at a given frequency. The following measurements are shown for the MSET corresponding to the third resonance in Fig. 3c (marked by a dashed rectangle), which was chosen due to its remarkable stability over time. Figure $4 \mathrm{a}$ shows $\overline{I_{\text {sd }}}$ as function of $V_{\text {sd }}$ for five different drive frequencies $f_{1}-f_{5}$ at $P_{\text {drive }}=29 \mathrm{dBm}$. Once again we have subtracted the offset of the current-voltage amplifier. For an actuation frequency of $f_{1}=3.804800 \mathrm{MHz}$, which is below the system's eigenfrequency, the mechanical amplitude of the MSET is insufficient to produce charge transport (black open squares). As the frequency is chosen to be closer to the MSET's eigenfrequency, a current develops. With increasing frequency the system moves through different oscillation regimes. This is characteristic for impacting oscillators, which move nonmonotonically through different oscillation regimes with changing system parameters, including 1-periodic, subharmonic and chaotic behaviour. For the MSET, 1-periodic oscillation gives rise to optimal charge transport, whereas subharmonic or chaotic behaviour limits the transport efficiency. Plots $f_{2}$ and $f_{3}$ in Fig. 4a illustrate how the system moves through different subharmonic oscillation regimes with increasing transport efficiency as $f_{\text {drive }}$ is incremented. The system eventually shows optimal transport in the limit of 1-periodic motion, for which the oscillation period is given by $T=1 / f_{\text {drive }}$ represented by $f_{4}$ and $f_{5}$. The island charges according to its capacitance $C_{\text {off }}$ (see Fig. 4b) when in contact with either source or drain, as the contact time $t_{0}$ between the island and either electrode is much larger than the RC time $\tau$ of the system. Therefore the charged island is in equilibrium and optimum shuttling in accordance with ref. 8 can be observed. For frequencies above $f_{5}$ the system eventually returns to the state of no transport when beyond resonance (not shown).

In the limit of 1-periodic motion $\left(f_{4}, f_{5}\right)$, the system can be modelled by the single-electron box ${ }^{17}$, which is schematically 

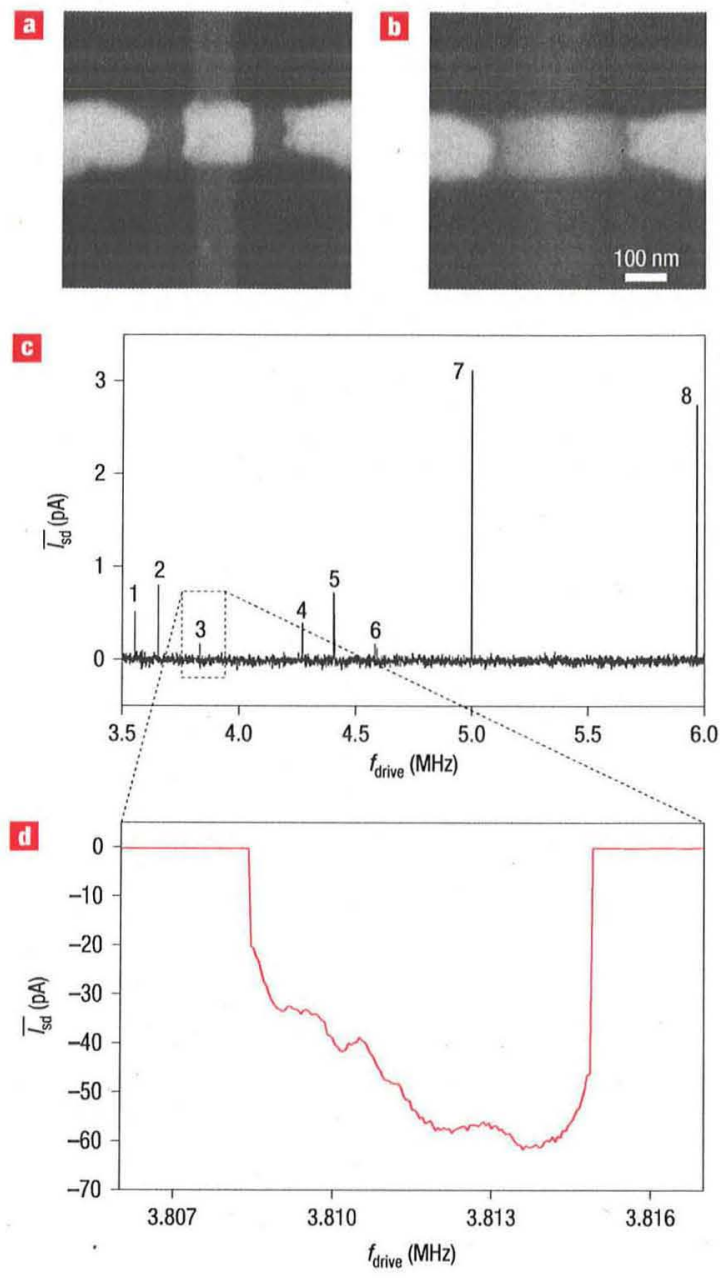

Figure 3 MSET resonances. a, SEM image of the gold island and parts of the source and drain electrodes of one MSET. b. SEM image of the gold island of the MSET oscillating between the source and drain electrodes while actuated by ultrasonic waves at the mechanical eigenfrequency. $\mathbf{c}$, Resonances of the MSET array observed in the electrical transport measurement at a temperature of approximately $20 \mathrm{~K}$. Each resonance corresponds to a different MSET of the array, which is mechanically excited at its eigenfrequency. The pressure is $7.5 \times 10^{-4} \mathrm{mbar}, P_{\text {drive }}=25 \mathrm{dBm}$ and $V_{\mathrm{sd}}=0.8 \mathrm{~V}$. d, High-resolution frequency sweep for the MSET corresponding to the third resonance in $\mathbf{c}$ at $P_{\text {drive }}=27.5 \mathrm{dBm}$ and $V_{\mathrm{sd}}=-2 \mathrm{~V}$. In contrast to $\mathrm{c}$, the resonance shows a negative amplitude because of the reversed source-drain voltage.

illustrated in Fig. 4b. We denote the capacitance of the tunnel junction between the island and the charging electrode as $C_{\text {on }}$, and refer to the capacitance of the island and the far electrode as $C_{\text {off }}$. From the model of the single-electron box, the electrostatic energy of the island is given by

$$
E_{\mathrm{ch}}\left(n, V_{\mathrm{sd}}\right)=\frac{\left(n e-V_{\mathrm{sd}} C_{\text {off }}\right)^{2}}{2\left(C_{\mathrm{on}}+C_{\text {off }}\right)}
$$

where $n$ is the number of excess charges on the island and $e$ is the elementary charge ${ }^{17}$. Combining the electrostatic energy with the

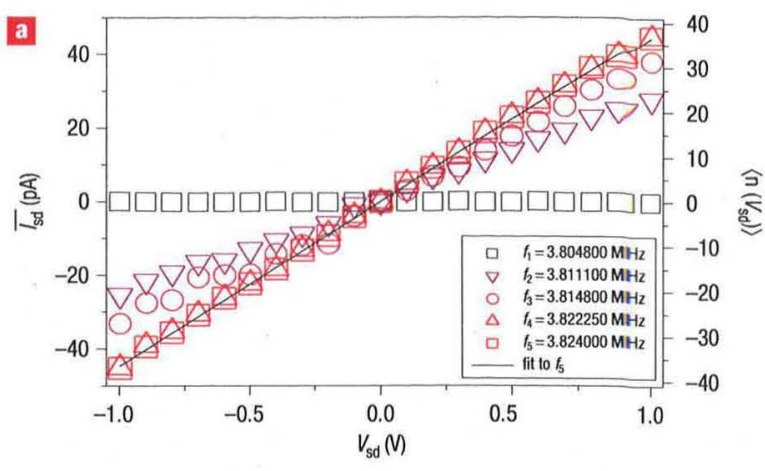

b
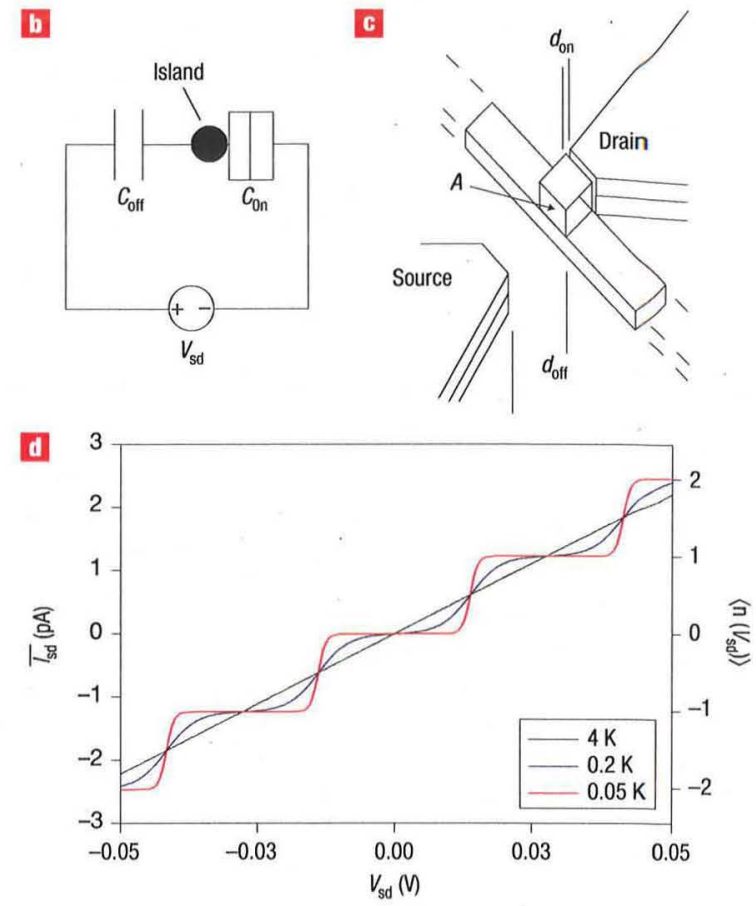

Figure 4 Measurement, model and simulation of the MSET. a, Plot showing the measured current $\bar{T}_{\mathrm{sd}}$ as a function of the source-drain voltage for five different actuation frequencies. The axis on the right-hand side of the graph shows the number of excess charges on the island, as defined in equation (2). b. Schematic of the single-electron box used as a model for the MSET. c. Graphical illustration of the parameters that are used in the parallel-plate capacitor model. d, Simulation of the current as a function of the source-drain voltage for the measured MSET at different temperatures. The $V_{\text {sd }}$ scale is magnified with respect to Fig. 4 a to resolve the emerging Coulomb staircase.

canonical probability distribution for the island excess charge $n$ yields the expectation value of the number of excess charges being transferred to the island:

$$
\left\langle n\left(V_{\mathrm{sd}}\right)\right\rangle=\frac{1}{Z_{\mathrm{ch}}} \sum_{n=-\infty}^{\infty} n \exp \left(-E_{\mathrm{ch}}\left(n, V_{\mathrm{sd}}\right) / k_{\mathrm{B}} T\right)
$$

where $Z_{\mathrm{ch}}$ is the partition function of the system, $k_{\mathrm{B}}$ is the Boltzmann constant and $T$ is the temperature ${ }^{17}$. In the event of charging, the number of charge carriers transferred to or from the island is twice the number of excess charges, accounting for 


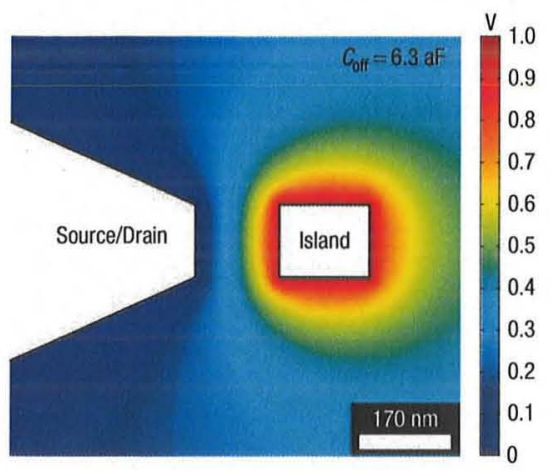

Figure 5 Finite element calculation. The electric potential for a voltage difference of $1 \mathrm{~V}$ between one side electrode and the gold island, determined by finite element calculations. The finite element simulation yields a capacitance $C_{\text {off }}=6.3 \mathrm{aF}$

holes and electrons ${ }^{9}$. Taking this into consideration we derive the following expression for the current:

$$
\overline{I_{\mathrm{sd}}}=2\left\langle n\left(V_{\mathrm{sd}}\right)\right\rangle f_{\mathrm{drive}} e
$$

Assuming a parallel plate capacitor model (Fig. 4c), we approximate $C_{\text {on }}=A \varepsilon_{0} / d_{\text {on }}$ and $C_{\text {off }}=A \varepsilon_{0} / d_{\text {off }}$ where $A$ is the cross-sectional area of the island facing the contact, $\varepsilon_{0}$ is the permittivity of free space, $d_{\mathrm{on}}$ is the distance between the island and the charging electrode, and $d_{\text {off }}$ is the distance between the island and the electrode not in contact, as illustrated in Fig. 4c. We take $d_{\text {on }}$ to be a typical tunnel distance of $1 \mathrm{~nm}$ (ref. 18). The distance between the island at rest and the electrodes is $80 \mathrm{~nm}$. From this we approximate the distance $d_{\text {off }}$ between the island and the electrode not in contact in the event of charging to be $160 \mathrm{~nm}$. Having assigned values for $d_{\text {on }}$ and $d_{\text {off }}$ equation (3) gives us an expression for the current with only one free parameter, the cross-sectional area $A$. We fit equation (3) to the curve $f_{5}$ of Fig. $4 \mathrm{a}$ in the limit of 1-periodic motion (solid line). For the fit we let the summation index $n$ run from $-1,000$ to 1,000 . We have run the fit for different values of $n$ to confirm that the fit result converges to one value. From $A$ obtained through the fit, we calculate $C_{\text {off }}$ to be $5.9 \mathrm{aF}$ and $C_{\text {on }}$ to be $950 \mathrm{aF}$. Figure $4 \mathrm{a}$ shows that the experimental data and the fit are in excellent agreement. We independently determine $C_{\text {off }}$ by finite element calculations using the program Femlab 3.3 (Comsol Multiphysics $\mathrm{GmbH}$ ). Figure 5 displays the electric potential between one side electrode and the island for an applied bias voltage of $1 \mathrm{~V}$, using our device dimensions. The simulation yields $C_{\text {off }}=6.3 \mathrm{aF}$, which agrees with the experimental result to an accuracy of $6 \%$.

Not only does this demonstrate that we achieve MSET operation as predicted by theory, but it also allows us to simulate the current-voltage dependence of our system for different temperatures, as illustrated in Fig. $4 \mathrm{~d}$. We find that the Coulomb blockade requirement $\Delta E_{\mathrm{ch}}=\left(e^{2} / 2\left(C_{\mathrm{on}}+C_{\text {off }}\right)\right) \gg k_{\mathrm{B}} T$ is satisfied for temperatures below $0.2 \mathrm{~K}$, which is shown by the evolving staircase shape of $\overline{I_{\mathrm{sd}}}$ in the simulation with bias voltages in the range of $20 \mathrm{mV}$. The second condition for Coulomb blockade is that the energy uncertainty given by the Heisenberg uncertainty relation due to the limited life time $\tau_{\text {sd }}$ of the island charge state is much smaller than the energy difference $\Delta E_{\text {ch }}$ between two subsequent charge states. Taking $\tau_{\text {sd }}$ to be the time the island travels between the two electrodes this requires for our system that

$$
f_{\text {drive }} \ll \frac{\Delta E_{\mathrm{ch}}}{h} \approx 1 \times 10^{10} \mathrm{~Hz}
$$

which is satisfied by several orders of magnitude. It has recently been demonstrated that the requirements for Coulomb blockade can be satisfied for a system with one mechanically modulated tunnel junction at a frequency as high as $82 \mathrm{MHz}$ (ref. 19), which exceeds our operation frequency by a factor of 10 . With this we conclude that the technology presented here paves the way for high-performance MSETs operated in the Coulomb blockade regime, which may have implications for quantum metrology.

\section{References}

1. Schoelkopf, R. J., Wahlgren, P., Kozhevnikov, A. A., Delsing, P. \& Prober, D. E. The radio-frequency single-electron transistor (RF-SET): A fast and ultrasensitive electrometer. Science 280 $1238-1242$ (1998)

2. Devoret, M. H. \& Schoelkopf, R. J. Amplifying quantum signals with the single-electron transistor. Nature 406, 1039-1046 (2000)

3. Keller, M. W., Martinis, J. M., Zimmermann, N. M. \& Steinbach, A. H. Accuracy of electron counting using 7-junction electron pump. Appl. Phys. Lett. 69, 1804-1806 (1996).

4. Ono, Y., Zimmermann, N. M., Yamazaki, K. \& Takahashi, Y. Turnstile operation using a silicon dualgate single-electron transitor. Jpn J. Appl. Phys. 42, L1109-L1111 (2003).

5. Geerligs, L. J. et al. Frequency-locked turnstile device for single electrons. Phys. Rev. Lett. 64 2691-2694 (1990).

6. Fonseca, L. R. C., Korotkov, A. N. \& Likharev, K. K. A numerical study of the accuracy of singleelectron current standards. J. Appl. Phys. 79, 9155-9165 (1996).

De Franceschi, S. et al. Electron cotunnelling in a semiconductor quantum dot. Phys. Rev. Lett. 86, 878-881 (2001).

8. Weiss, C. \& Zwerger, W. Accuracy of a mechanical single-electron shuttle. Europhys. Lett. 47, 97-103 (1999).

Gorelik, L. Y et al. Shuttle mechanism for charge transfer in coulomb blockade nanostructures. Plys. Rev. Lett. 80, 4526-4529 (1998).

0. Erbe, A., Blick, R. H., Tilke, A., Kriele, A. \& Kotthaus, J. P. A mechanically flexible tunneling contact operating at radio frequencies. Appl. Phys. Lett. 73, 3751-3753 (1998).

operating at radio frequencies. Appl. Phys. Lett. 73, 3751-3753 (1998). . Erbe, A., Weiss, C., Zwerger, W. Q Blick, R. H. Nanom

radio fequencies. Phys. Kev. Letl. 87, 006106 (2001). 2. Scheible, D. V. \& Blick., R. H. Sil

13. Verbridge, S. S., Parpia, J. M., Reichenbach, R. B., Bellan, L. M. \& Craighead, H. G. High quality factor resonance at room temperature with nanostrings under high tensile stress. J. Appl. Phys. 99 $124304(2006)$

14. Jänchen, G. et al. Mechanical properties of high-aspect-ratio atomic-force microscope tips. App Phys. Lett. 80, 4623-4625 (2002).

15. Shaw, S. W. The dynamics of a harmonically excited system having rigid amplitude constraints. J. Appl. Mech. 52, 453-464 (1985).

16. Isacsson, A. Dynamics of a three-terminal mechanically flexible tunnelling contact. Phys. Rev. B 64, 035326 (2001)

17. Devoret, M. H. \& Grabert, H. in Single Charge Timneling (eds Grabert, H. \& Devoret, M. H.) (Plenum, New York, 1992)

18. Sze, S. M. in Physics of Semiconductor Devices (ed. Sze, S. M.) (John Wiley \& Sons, New York, 1981). 19. Azuma, Y. et al. One by one single-electron transport in nanomechanical Coulomb blockade shuttle. Appl. Plys. Lett. 91, 053120 (2007).

\section{Acknowledgements}

We gratefully acknowledge financial support of the German Excellence Initiative via the Nanosystem Initiative Munich (NIM) and by the Deutsche Forschungsgemeinschaft (Ko 416/18-1). We thank K. Karrai for helpful discussion and S. Manus for expert technical help. D.R.K. thankfully acknowledges support by the Studienstiftung des deutschen Volkes.

\section{Author contributions}

D.R.K. conceived and designed the experiment, analysed the data and wrote the paper. E.M.W. and J.P.K. co-wrote the paper. All authors discussed the results and commented on the manuscript. 